\title{
UNRAMIFIED WHITTAKER FUNCTIONS ON THE METAPLECTIC GROUP
}

\author{
YUVAL Z. FLICKER
}

(Communicated by Bhama Srinivasan)

\begin{abstract}
Kazhdan (unpublished), Shintani [Sh] and Casselman and Shalika [CS] computed explicitly the unramified Whittaker function of a quasisplit $p$-adic group. This is the main local ingredient used in the RankinSelberg-Shimura method, which yielded interesting results in the study of Euler products such as $L\left(s, \pi \otimes \pi^{\prime}\right)$ by Jacquet and Shalika [JS] (here $\pi, \pi^{\prime}$ are cuspidal $G L\left(n, A_{F}\right)$-modules), and $L(s, \pi, r)$ by [F] (here $\pi$ is a cuspidal $G L\left(n, A_{E}\right)$-module, $E$ is a quadratic extension of the global field $F$, and $r$ is the twisted tensor representation of the dual group of $\operatorname{Res}_{E / F} G L(n)$ ). Our purpose here is to generalize Shintani's computation [Sh] from the context of $G L(n)$ to that of the metaplectic $r$-fold covering group $\tilde{G}$ of $G L(n)$ (see $\left[\mathbf{F}^{\prime}\right.$, $\mathbf{F K}]$ ).
\end{abstract}

Notations. Let $F$ be a nonarchimedean local field with a ring $R$ of integers and a uniformizer $u$ of the maximal ideal of $R$. Denote by $q$ the cardinality of the residue field $R /(u)$ of $F$. Let $r, n$ be positive integers. Put $G=G L(n, F), K=G L(n, R)$. Let $\mu_{r}$ be the cyclic group of order $r$. Denote by $\tilde{G}$ the $r$-fold central topological covering group of $G$ (see [FK]). Then there is an exact sequence $1 \rightarrow \mu_{r} \stackrel{i}{\rightarrow} \tilde{G} \stackrel{p}{\rightarrow}$ $G \rightarrow 1$, with a preferred section $s: G \rightarrow \tilde{G}$ of $p$. We identify $\mu_{r}$ with its image via $i$. We also fix an embedding of $\mu_{r}$ in the field $\mathbf{C}$ of complex numbers. Suppose that $r$ is a unit in $R$ (its valuation is one). Then $K$ embeds (see [FK]) as a subgroup of $\tilde{G}$; we identify $K$ with its image. Fix a Haar measure on $G$ by the requirement that the volume of $K$ is one.

Let $L_{c}(\tilde{G} / / K)$ denote the commutative convolution algebra (see $[\mathbf{F K}]$ ) of complex-valued compactly-supported $K$-biinvariant anti-genuine functions on $\tilde{G}$. A function $f: \tilde{G} \rightarrow \mathbf{C}$ is called anti-genuine if $f(\varsigma g)=\varsigma^{-1} f(g)$ for all $\zeta$ in $\mu_{r}$ and $g$ in $\tilde{G}$. In writing $\varsigma g$ we used the embedding of $\mu_{r}$ in $\tilde{G}$; in writing $\varsigma^{-1} f(g)$ we used the embedding of $\mu_{r}$ in $\mathbf{C}^{\times}$. Let $\pi$ be an irreducible representation of $\tilde{G}$ which is unramified (has a nonzero $K$-fixed vector) and genuine $\left(\pi(\varsigma g)=\varsigma \pi(g) ; \varsigma\right.$ in $\mu_{r}, g$ in $\tilde{G}$ ). By the theory of the Satake transform (see $[\mathbf{F K}]$ ) it determines an algebra homomorphism, denoted again by $\pi$, of $L_{c}(\tilde{G} / / K)$ into $\mathbf{C}$.

For any $n$-tuple $m=\left(m_{1}, \ldots, m_{n}\right)$ of integers, denote by $u^{m}$ the diagonal matrix whose $i$ th diagonal entry is $u^{m_{i}}(1 \leq i \leq n)$. Denote by $N$ the group of upper triangular unipotent matrices in $G$. The section $s$ injects $N$ and $u^{r \mathbf{Z}}$ as subgroups of $\tilde{G}$ (see [FK]). We identify $N$ and $u^{r \mathbf{Z}}$ with their images in $\tilde{G}$. Write $m(i)$ for

Received by the editors September 16, 1986.

1980 Mathematics Subject Classification (1985 Revision). Primary 11F85; Secondary 11F27, 11F37, 11F70.

Partially supported by an NSF grant. 
$m=(1, \ldots, 1,0, \ldots, 0)$ where 1 appears in the first $i$ places. Let $f_{i}(1 \leq i \leq n)$ be the member of $L_{c}(\tilde{G} / / K)$ which is supported on $\mu_{r} K u^{r m(i)} K$ and attains the value 1 at $u^{r m(i)}$. Then $L_{c}(\tilde{G} / / K)$ is isomorphic to the polynomial ring generated by $f_{1}, \ldots, f_{n}$.

Choose $n$ complex numbers $t_{1}, \ldots, t_{n}$ so that the $i$ th elementary symmetric function $\operatorname{Sym}_{i}\left(\left(t_{j}\right)\right)=\sum_{j_{1} \leq \cdots \leq j_{i}} t_{j_{1}} \cdots t_{j_{i}}$ in the $t_{j}$ 's is equal to $q^{i r(i-1) / 2} \pi\left(f_{i}\right)$ $(1 \leq i \leq n)$. Let $t$ be the diagonal matrix whose $i$ th diagonal entry is $t_{i}(1 \leq i \leq n)$. It lies in $G L(n, \mathbf{C})$ since $\operatorname{det} t=q^{n r(n-1) / 2} \pi\left(f_{n}\right) \neq 0$.

Let $c(t)$ be the complex-valued function on $\mathbf{Z}^{n}$ which attains the value zero at $m=\left(m_{1}, \ldots, m_{n}\right)$ unless $m_{1} \geq \cdots \geq m_{n}$, where

$$
c(t ; m)=\operatorname{det}\left(t_{i}^{m_{j}+n-j} ; 1 \leq i, j \leq n\right) / \operatorname{det}\left(t_{i}^{n-j} ; 1 \leq i, j \leq n\right) .
$$

Here the numerator is called a Schur function (see [M, p. 24]), and it is divisible in $\mathbf{Z}\left[t_{1}, \ldots, t_{n}\right]$ by the denominator, which is the Vandermonde determinant $\prod\left(t_{i}-t_{j}\right)(1 \leq i<j \leq n)$. Note that for $m$ with $m_{1} \geq \cdots \geq m_{n}, c(t ; m)$ is the value at $t$ of the character of the irreducible representation of $G L(n, \mathbf{C})$ with highest weight $m$. We have $c(t ; m(i))=\operatorname{Sym}_{i}\left(\left(t_{j}\right)\right)$, and this is equal to $q^{i r(i-1) / 2} \pi\left(f_{i}\right)$ by the definition of $t$.

Choose a character $\psi$ of the additive group of $F$ which is trivial on $R$ but not on $u^{-1} R$. Denote by $\psi$ also the character of $N$ given by $\psi(x)=\prod_{i=1}^{n-1} \psi\left(x_{i, i+1}\right)$, where $x_{i, i+1}$ is the $(i, i+1)$ entry of $x$. Given $\pi$ and $\psi$, the function $W$ on $\tilde{G}$ is called an unramified Whittaker function associated with $\pi$ and $\psi$ if it satisfies

$$
W(\varsigma x g k)=\varsigma \psi(x) W(g) \quad\left(x \text { in } N, \varsigma \text { in } \mu_{r}, g \text { in } \tilde{G}, k \text { in } K\right),
$$

and

$$
\pi(f) W(g)=\int_{G} W(g h) f(h) d h \quad\left(f \text { in } L_{c}(\tilde{G} / / K), g \text { in } \tilde{G}\right) .
$$

The integral is taken over $G \simeq \tilde{G} / \mu_{r}$; the integrand is invariant under $\mu_{r}$. Let $D$ be the set of $m$ with $m_{1} \geq \cdots \geq m_{n} \geq 0$ and $r+m_{i}<m_{i+1}(1 \leq i<n)$. For each $d$ in $D$ put $a(d ; m)=W\left(s\left(u^{d}\right) u^{r m}\right)$. Recall that we identify $u^{r \overline{\mathbf{Z}}}$ with its image in $\tilde{G}$ via the section $s$. Since $G=\bigcup N u^{m} K$ (disjoint union over $m$ in $\mathbf{Z}^{n}$ ), to determine $W$ on $\tilde{G}$ it suffices (by (1)) to evaluate $a(d ; m)$ for all $d$ in $D$ and $m$ in $\mathbf{Z}^{n}$. Since the conductor of $\psi$ is $R$, if follows from (1) that $a(d ; m)$ is zero unless $m_{1} \geq m_{2} \geq \cdots \geq m_{n}$.

THEOREM. For each $d$ in $D$ we have $a(d ; m)=a(d ; 0) q^{r \sum_{i=1}^{n}(i-n) m_{i}} c(t ; m)$ for all $m$ in $\mathbf{Z}^{n}$.

Let $I(i)$ be the set of all $n$-tuples $e=\left(e_{1}, \ldots, e_{n}\right)$ with entries $e_{i}$ equal to zero or one such that $e_{1}+\cdots+e_{n}=i$. Put $N_{R}=N \cap K$ and $N_{R}(e)=N_{R} \cap u^{e} K u^{-e}$. Note that the cardinality $\left[N_{R} / N_{r}(r e)\right]$ of $N_{R} / N_{R}(r e)$ is $q^{r w}$, where

$$
w=\sum_{j>k} \max \left(e_{j}-e_{k}, 0\right)=i n-i(i-1) / 2-\sum_{j=1}^{n} j e_{j} .
$$

Denote by $f\left[x u^{r e} K\right]$ the right $K$-invariant anti-genuine complex-valued function on $\tilde{G}$ which is supported on $\mu_{r} x u^{r e} K\left(e\right.$ in $I(i), x$ in $\left.N_{R} / N_{R}(r e)\right)$, and attains the value one at $x u^{r e}$.

We first assume the validity of the following lemma. 
LEMMA. For each $1 \leq i \leq n$ we have $f_{i}=\sum_{e \in I(i)} \sum_{x \in N_{R} / N_{R}(r e)} f\left[x u^{r e} K\right]$. have

The Lemma implies that for $m=\left(m_{1}, \ldots, m_{n}\right)$ in $\mathbf{Z}^{n}$ with $m_{1} \geq \cdots \geq m_{n}$ we

$$
\begin{aligned}
\pi\left(f_{i}\right) a(d ; m) & =\int_{G} W\left(s\left(u^{d}\right) u^{r m} g\right) f_{i}(g) d g \\
& =\sum_{e}\left[N_{R} / N_{R}(r e)\right] a(d ; m+e) \quad(e \text { in } I(i)) \\
& =q^{i r n-i r(i-1) / 2} \sum_{e} q^{-r \sum_{j=1}^{n} j e_{j}} a(d ; m+e) .
\end{aligned}
$$

Put

$$
b(d ; m)=q^{r \sum_{j=1}^{n}(n-j) m_{j}} a(d ; m) .
$$

If $m_{1} \geq \cdots \geq m_{n}$, then we have

$$
q^{i r(i-1) / 2} \pi\left(f_{i}\right) b(d ; m)=\sum_{e} b(d ; m+e) \quad(1 \leq i \leq n) .
$$

Otherwise $b(d ; m)=0$. Namely $b(d ; m)$ satisfies the equation

$$
c(t ; m(i)) b(d ; m)=\sum_{e} b(d ; m+e) \quad \text { if } m_{1} \geq \cdots \geq m_{n} .
$$

On the other hand, the function $c(t ; j)$ satisfies the equation

$$
c(t ; m(i)) c(t ; m)=\sum_{e} c(t ; m+e) \quad \text { if } m_{1} \geq \cdots \geq m_{n} .
$$

Hence both $c(t ; m)$ and $b(d ; m)$ (for each $d$ in $D$ ) are functions of $m$ in $\mathbf{Z}^{n}$ which satisfy the same system of difference equations which has a unique solution up to a constant multiple. Since $c(t ; 0)=1$ the theorem follows.

It remains to prove the Lemma.

ProOF OF LEMMA. Let $W$ be the Weyl group of permutation matrices in $K$, realized as a group of matrices with entries zero and one only. Let $I$ be the Iwahori subgroup of $K$ which consists of all matrices in $K$ whose under diagonal entries are all in $u R$. Put $\bar{N}$ for the group of lower triangular unipotent matrices, $N_{I}$ for $\bar{N} \cap I$, and $A_{R}$ for the diagonal subgroup of $K$. We have the decompositions $I=N_{R} A_{R} \bar{N}_{I}=\bar{N}_{I} A_{R} N_{R}$ and $K=\bigcup N_{R} \bar{N}_{I} w N_{R} A_{R}$ (disjoint union over $w$ in $W)$. Put $e(i)$ for $m=(0, \ldots, 0,1, \ldots, 1)$, where 1 appears in the last $i$ entries. Since $u^{-r e(i)} N_{R} u^{r e(i)}$ lies in $K$, we have

$$
K u^{r m(i)} K=K u^{r e(i)} K \subset \bigcup_{w \in W} N_{R} A_{R} \bar{N}_{I} w u^{r e(i)} w^{-1} K .
$$

Put $y$ for the element $w u^{r e(i)} w^{-1}$ of $\tilde{G}$. For $1 \leq j<k \leq n$, and a matrix $\left(\begin{array}{ll}a & b \\ c & d\end{array}\right)$, write $\left(\begin{array}{ll}a & b \\ c & d\end{array}\right)_{j k}$ for the matrix $\left(x_{u v}\right)$ in $G L(n)$ whose entries along the diagonal are one except that $x_{j j}=a, x_{k k}=d$, and its nondiagonal entries are zero except that $x_{j k}=b$ and $x_{k j}=c$.

Suppose that $\bar{n}=\left(\begin{array}{ll}1 & 0 \\ x & 1\end{array}\right)_{j k}$ is a matrix in $\bar{N}_{I}$, and $y^{-1} \bar{n} y$ does not lie in $K$. Write $|\cdot|$ for the valuation on $F$ normalized by $|u|=q^{-1}$. Then $q^{-r}<|x|<1$, the 
$j$ th diagonal entry of $p(y)$ is one and the $k$ th is $u^{r}$, and

$$
y^{-1} \bar{n} y=\left(\begin{array}{cc}
1 & 0 \\
u^{-r} x & 1
\end{array}\right)_{j k} \text {. }
$$

We have

$$
\left(\begin{array}{ll}
1 & 0 \\
x & 1
\end{array}\right)_{j k}=\left(\begin{array}{cc}
1 & x^{-1} \\
0 & 1
\end{array}\right)_{j k}\left(\begin{array}{cc}
0 & -1 \\
1 & 0
\end{array}\right)_{j k}\left(\begin{array}{cc}
x & 0 \\
0 & x^{-1}
\end{array}\right)_{j k}\left(\begin{array}{cc}
1 & x^{-1} \\
0 & 1
\end{array}\right)_{j k} .
$$

Given the matrix $s\left(\left(\begin{array}{ll}a & 0 \\ 0 & b\end{array}\right)_{j k}\right)$ in $\tilde{G}$, we have in $\tilde{G}$ (see [FK, (2.1)]) the relation

$$
\begin{gathered}
s\left(\left(\begin{array}{ll}
a & 0 \\
0 & b
\end{array}\right)_{j k}\right) s\left(\left(\begin{array}{cc}
x & 0 \\
0 & x^{-1}
\end{array}\right)_{j k}\right) s\left(\left(\begin{array}{ll}
a & 0 \\
0 & b
\end{array}\right)_{j k}\right)^{-1} \\
=s\left(\left(\begin{array}{cc}
x & 0 \\
0 & x^{-1}
\end{array}\right)_{j k}\right)\left(\frac{b}{a, x}\right) .
\end{gathered}
$$

Here $(\cdot, \cdot)$ denotes the nondegenerate bimultiplicative $r$ th Hilbert symbol

$$
F^{\times} / F^{\times r} \times F^{\times} / F^{\times r} \rightarrow \mu_{r} .
$$

Taking $b=1$ and a unit $a$, namely $\left(\begin{array}{ll}a & 0 \\ 0 & 1\end{array}\right)_{j k}$ in $K$, we conclude that the anti-genuine $K$-biinvariant function $f_{i}$ attains the value zero at $\bar{n} y$, since

$$
\begin{aligned}
f_{i}(\bar{n} y)= & f_{i}\left(y s\left(\left(\begin{array}{cc}
1 & x^{-1} u^{r} \\
0 & 1
\end{array}\right)_{j k}\right) s\left(\left(\begin{array}{cc}
0 & -1 \\
1 & 0
\end{array}\right)_{j k}\right)\right. \\
& \left.\times s\left(\left(\begin{array}{cc}
x u^{-r} & 0 \\
0 & x^{-1} u^{r}
\end{array}\right)_{j k}\right) s\left(\left(\begin{array}{cc}
1 & x^{-1} u^{r} \\
0 & 1
\end{array}\right)_{j k}\right)\right) \\
= & f_{i}\left(s\left(\left(\begin{array}{cc}
x u^{-r} & 0 \\
0 & x^{-1} u^{r}
\end{array}\right)_{j k}\right) y\right) \\
= & f_{i}\left(s\left(\left(\begin{array}{cc}
a & 0 \\
0 & 1
\end{array}\right)_{j k}\right) s\left(\left(\begin{array}{cc}
x u^{-r} & 0 \\
0 & \left.x^{-1} u^{r}\right)_{j k}
\end{array}\right) y\right)^{\prime}\right) \\
= & \left.\left.(a, x) f_{i}\left(\begin{array}{cc}
s\left(\left(\begin{array}{cc}
x u^{-r} & 0 \\
0 & x^{-1} u^{r}
\end{array}\right)_{j k}\right.
\end{array}\right) y s\left(\begin{array}{cc}
a & 0 \\
0 & 1
\end{array}\right)_{j k}\right)\right) \\
= & \left.(a, x) f_{i}\left(\begin{array}{cc}
s\left(\left(\begin{array}{cc}
x u^{-r} & 0 \\
0 & x^{-1} u^{r}
\end{array}\right)_{j k}\right.
\end{array}\right) y\right) \\
= & (a, x) f_{i}(\bar{n} y) .
\end{aligned}
$$

Since the Hilbert symbol $(\cdot, \cdot)$ is nondegenerate we can find a unit $a$ with $(a, x) \neq 1$; indeed, $(a, b)=1$ for any pair $a, b$ of units, hence there exists some unit $a$ with $(a, u)=\varsigma$, where $\varsigma$ is a primitive $r$ th root of unity. It follows that $f_{i}$ is supported on the subset

$$
\mu_{r} \bigcup_{w \in W} N_{R} w u^{r e(i)} w^{-1} K \quad \text { of } \tilde{G} .
$$

Since this set is contained in $\mu_{r} K u^{m(i)} K$, the Lemma follows. 
REMARK. If $\pi$ has a Whittaker model, namely we have $\pi(g) W(h)=W(h g)$ for all $h, g$ in $\tilde{G}$, then $a(d ; m)=\pi\left(s\left(u^{d}\right) u^{r m}\right) a(0 ; 0)$, and in particular $a(d ; 0)=$ $\pi\left(s\left(u^{d}\right)\right) a(0 ; 0)$ for all $d$ in $D$. In this case there exists a unique (up to a scalar multiple) unramified Whittaker function associated with $\pi, \psi$.

\section{REFERENCES}

[CS] W. Casselman and J. Shalika, The unramified principal series of p-adic groups. II: The Whittaker function, Compositio Math. 41 (1980), 207-231.

[F] Y. Flicker, Twisted tensor and Euler products, Bull. Soc. Math. France (1987/8).

[F'] , Automorphic forms on covering groups of $G L(2)$, Invent. Math. 57 (1980), 119-182.

[FK] Y. Flicker and D. Kazhdan, Metaplectic correspondence, Publ. Math. Inst. Hautes Études Sci. 64 (1987), 53-110.

[JS] H. Jacquet and J. Shalika, On Euler products and the classification of automorphic representations. I, Amer. J. Math. 103 (1981), 499-558.

[M] I. MacDonald, Symmetric functions and Hall polynomials, Clarendon Press, Oxford, 1979.

[Sh] T. Shintani, On an explicit formula for class-1 "Whittaker functions" on $G L_{n}$ over p-adic fields, Proc. Japan Acad. 52 (1976), 180-182.

Department of Mathematics, HaRvard University, SCIEnCE Center, ONe OXFORD STREET, CAMBRIDGE, MASSACHUSETTS 02138 